\title{
Diversidade de macrofauna edáfica em diferentes ambientes de cultivo no agreste da Paraíba, Brasil
}

\author{
José Thyago Aires Souza ${ }^{1}$, Suenildo Josemo Costa Oliveira ${ }^{2}$, Fábio Agra de Medeiros \\ Nápoles $^{2}$, Mileny dos Santos de Souza ${ }^{1}$, Marcio Raimundo de Medeiros ${ }^{3}$ \\ ${ }^{1}$ Universidade Federal da Paraíba, Campus Areia, Areia-PB, Brasil. E-mail: thyago.agro@ hotmail.com, mileny.lopes67@gmail.com \\ 2 Universidade Estadual da Paraíba, Campus Lagoa Seca, Lagoa Seca-PB, Brasil. E-mail: suenildo@ccaa.uepb.edu.br, \\ fnapoles@uol.com.br \\ ${ }^{3}$ Empresa Brasileira de Pesquisa Agropecuária do Rio Grande do Norte, Base Física de Cruzeta, Cruzeta-RN, Brasil. E-mail: \\ marcioemparn@hotmail.com
}

Recebido: 21/05/2017; Aceito: 20/07/2017

\section{RESUMO}

A diversidade da fauna edáfica tem sido indicadora da qualidade do solo em ambientes explorados para atividades agrícolas com constante interferência antrópica; tem se tornado um fator essencial para a manutenção da estrutura e fertilidade edáfica. Objetivou-se identificar a população de macrofauna presente em solo cultivado com as culturas do pinhão manso (Jatropha curcas L.) e laranjeira (Citrus sinensis L.). Foram selecionados sete pontos de coletas fixos onde utilizou-se armadilhas do tipo Provid, enterradas com os bordos das quatro aberturas ao nível da superfície do solo. Após a quantificação dos indivíduos, calculou-se a riqueza ( $\mathrm{n}^{\circ}$ de grupos identificados), os índices de frequência, abundância, dominância e constância, obtidos através programa ANAFAU e também os índices de diversidade de Shannon $(\mathrm{H})$ e uniformidade de Pielou (e); os dados foram submetidos à análise de variância e as médias comparadas pelo teste de Tukey a 5\% e 1\% de probabilidade. As ordens da macrofauna mais abundantes que apresentaram maior frequência e constância seguida por menores índices de Shannon e de Pielou foram: Hymenoptera, Araneae e Coleoptera, respectivamente. O solo cultivado com Citrus sinensis L. apresentou maior abundância e diversidade de ordens de indivíduos da macrofauna edáfica.

Palavras-chave: Fauna do solo, indicadores de fertilidade, Citrus sinensis, Jatropha curcas.

\section{Diversity of edaphic macrofauna in different cropping environments in the agreste of Paraíba, Brazil}

\begin{abstract}
The diversity of soil fauna as an indicator of soil quality in environments exploited for agricultural activities, where there is constant anthropic interference has become an essential factor for the maintenance of soil structure and fertility. The objective of this study was to identify the macrofauna population present in soil cultivated with Jatropha curcas L. and orange tree (Citrus sinensis L.). Seven fixed collection points were selected, where traps of the Provid type were used, buried with the edges of the four openings at the level of the soil surface. After quantification of the individuals, the richness (number of groups identified), frequency, abundance, dominance and constancy indexes were calculated, obtained through ANAFAU program and, also the diversity indexes of Shannon $(\mathrm{H})$ and uniformity of Pielou (e), the data were submitted to analysis of variance and the averages compared by the Tukey test at $5 \%$ and $1 \%$ probability. The more abundant macrofauna orders, which presented greater frequency and constancy, followed by lower indices of Shannon and Pielou were: Hymenoptera, Araneae and Coleoptera, respectively. Soil cultivated with Citrus sinensis L. showed higher abundance and diversity of orders of individuals of edaphic macrofauna.
\end{abstract}

Key words: Soil fauna; Fertility indicators; Citrus sinensis; Jatropha curcas. 


\section{Introdução}

Os microrganismos e animais invertebrados existentes no sistema solo, denominados de biota do solo, apresentam grande variedade de espécies, tamanhos e metabolismos e são responsáveis por inúmeras funções no ambiente edáfico. Dentre os invertebrados do solo, a macrofauna é fundamental ao funcionamento do ecossistema pois, além de afetar a produção primária de maneira direta e indireta, ocupa todos os níveis tróficos na cadeia alimentar do solo (SILVA et al., 2007; ALMEIDA et al., 2015).

As práticas agrícolas podem alterar a composição e diversidade dos organismos edáficos em diferentes graus de intensidade, por meio das mudanças de habitat, fornecimento de alimentos, criação de microambientes e competição intraespecífica e interespecífica (MARQUES et al., 2014; TERRY et al., 2015). Em ambientes explorados com atividades agrícolas onde constantemente há interferência antrópica, a utilização da fauna edáfica como indicadora da qualidade do solo é fundamental (SILVA et al., 2014).

Para Souza et al. (2016), informações sobre a biota do solo em áreas implantadas com diferentes culturas podem subsidiar o planejamento para a melhoria das características edáficas, pois além de avaliar as transformações exercidas pela intervenção antrópica, o estudo da macrofauna edáfica serve como monitoramento das modificações nestes ambientes fornecendo também, informações sobre a conservação e manutenção do equilíbrio dos agroecossistemas.

O pinhão manso (Jatropha curcas L.), oleaginosa que apresenta boa adaptação ás condições edafoclimáticas do Semiárido Brasileiro, podendo ser cultivada até em condições de sequeiro tem se tornado boa alternativa para o agricultor familiar tanto para extração de óleo vegetal quanto para outras finalidades (NÁPOLES et al., 2016). A laranjeira (Citrus sinensis L.) há tempos têm se destacado por promover o crescimento sócio econômico, contribuindo com a balança comercial nacional e principalmente, por gerar direta e indiretamente empregos na área rural. Boa parte da produção desta citrícola é consumida "in natura", porém, 50 a $55 \%$ é industrializada para a produção de suco (LOPES et al., 2011).

Desta forma, este trabalho objetivou determinar a composição da macrofauna invertebrada do solo em dois ambientes distintos, cultivados com pinhão manso (Jatropha curcas L.) e laranjeira (Citrus sinensis L.) no agreste da Paraíba.

\section{Material e Métodos}

O estudo foi realizado no município da Lagoa Seca, localizado na mesorregião do agreste paraibano cujo clima é tropical úmido, com precipitação média anual de $990 \mathrm{~mm}$ e umidade relativa do ar média de $60 \%$ (CPRM, 2005). A área cultivada com pinhão manso localiza-se no Campus II da Universidade Estadual da Paraíba e o pomar de laranjeiras pertence a uma propriedade vizinha, conhecida como Lagoa do Gravatá localizada à poucos metros do Campus Universitário no município de Lagoa Seca.

Para a coleta dos indivíduos da macrofauna foi adotado o método de captura do tipo Provid (ANTONIOLLI et al., 2006), constituído por recipientes PET transparentes com capacidade de $2 \mathrm{~L}$ com quatro aberturas de aproximadamente $2 \times 2 \mathrm{~cm}$ a $20 \mathrm{~cm}$ da base e preenchidos cada um com $200 \mathrm{ml}$ de uma solução aquosa de detergente neutro a $10 \%$ e cinco gotas de formaldeído a $20 \%$ (ambos com o objetivo respectivo de capturar e conservar os espécimes).

Para cada área foram utilizadas 10 armadilhas enterradas de forma casualizada no solo da área experimental até o limite inferior das aberturas e coletadas a cada 7 dias por um de período de quatro semanas; em seguida seu conteúdo foi peneirado e acondicionado em frascos contendo solução de álcool a $70 \%$ até o momento da identificação. A classificação dos organismos foi realizada através de lupa binocular no Laboratório de Microbiologia da Universidade Estadual da Paraíba, Campus II, Lagoa Seca, PB, onde foi realizada a contagem dos indivíduos e a identificação dos organismos da ordem dos grandes grupos taxonômicos.

Os experimentos foram implantados simultaneamente no mês de abril de 2013, época chuvosa. Segundo Lima et al. (2010) e Souza et al. (2016) há um aumento na macrofauna edáfica neste período; Lavelle (1988) afirma que em regiões tropicais a macrofauna do solo deve ser amostrada durante e até o final da estação chuvosa quando os fatores climáticos temperatura e umidade ainda não são limitantes.

O delineamento experimental adotado foi inteiramente casualizado, cujos tratamentos corresponderam às áreas de cultivo de Jatropha curcas L. e Citrus sinensis L. com dez repetições. Na avaliação do comportamento ecológico da macrofauna foram mensurados além do número total de indivíduos, os índices de frequência, abundância, dominância e constância, obtidos através software ANAFAU (SILVEIRA NETO et al., 2005). Determina-se aqui o conceito de: Frequência (F) - porcentagem de indivíduos de uma espécie em relação ao total de insetos coletados, classificando-se como: Pouco frequente (pf), Frequente (f), Muito frequente (mf). Abundância (A) refere-se ao número de indivíduos por unidade de superfície ou volume e varia no espaço e no tempo, sendo estabelecidas as seguintes classes de abundância: Rara (r), Dispersa (d), Comum (c), Abundante (a) e Muito abundante (ma); Dominância (D) - ação exercida 
pelos organismos dominantes de uma comunidade. A dominância, analisada pelo método de Laroca e Mielke (1975), consiste na capacidade da espécie em modificar, em seu benefício, o impacto recebido do ambiente, podendo assim causar o aparecimento ou desaparecimento de outros organismos; Constância (C) - porcentagem de espécies presentes nos levantamentos efetuados. As espécies foram separadas em categorias, segundo a classificação proposta por Bodenheimer (1955): a) Espécies constantes $(\mathrm{w})=$ presentes em mais de 50\% das coletas; b) Espécies acessórias (y) = presentes em 25-50\% das coletas; c) Espécies acidentais (z) $=$ presentes em menos de $25 \%$ das coletas.

A partir daí, calculou-se o índice de diversidade de Shannon $(\mathrm{H})(\mathrm{H}=-\Sigma$ pi. $\log$ pi, onde pi $=$ ni $/ \mathrm{N}, \mathrm{ni}=$ densidade de cada grupo e $\mathrm{N}=\Sigma$ da densidade de todos os grupos) com resultados variando de zero a cinco, cujos menores valores indicam menor diversidade e correspondente maior dominância de um ou mais grupos na comunidade (PASQUALIN et al., 2012; ALMEIDA et al., 2015). Foi calculado também o índice de uniformidade de Pielou (e) (e = H/log $\mathrm{S}$, com $\mathrm{H}=$ índice de Shannon e $\mathrm{S}$ = número de grupos), que varia de zero a um, com o valor mais elevado indicando maior homogeneidade e, portanto, máxima diversidade, uma vez que todos os grupos são igualmente abundantes (RICKLEFS, 2003).

Os dados foram submetidos à análise de variância (teste F) e as médias comparadas pelo teste de Tukey a $5 \%$ de probabilidade, utilizando através do software estatístico SISVAR-ESAL (FERREIRA, 2003).

\section{Resultados e Discussão}

Durante o período experimental foram identificados 102 indivíduos no solo cultivado com pinhão manso (Jatropha curcas L.), pertencentes à 8 ordens, enquanto que sob o cultivo de Citrus sinensis foram amostrados 201 indivíduos de 9 ordens; em ambos os casos as ordens pertenciam a 2 classes, Arachnida e Insecta (Tabela 1). Dentre as ordens identificadas, as que obtiveram o maior quantitativo de indivíduos foram Hymenoptera (78 e 143) e Araneae (7 e 17) no solo cultivado com Jatropha curcas e Citrus sinensis, respectivamente.

Estes resultados diferem dos observados por Souza et al., (2016) em trabalho realizado no Cariri do estado da Paraíba, onde as ordens Hymenoptera e Coleoptera foram as mais abundantes no cultivo de Cocos nucifera L., Vigna unguiculata L. e Opuntia ficus indica Mill. Entretanto, Correia et al. (2009) analisando a macrofauna edáfica no município de Lagoa Seca-PB em áreas cultivadas com acerola (Malpighia emarginata
DC.), mandioca (Manihot esculenta) e uma área de borda de mata observaram predominância das ordens hymenoptera e araneae assim como observado neste trabalho.

Tabela 1. Número e de indivíduos da macrofauna coletados em área com pinhão manso (Jatropha curcas L.) e laranjeira (Citrus sinensis L.)

\begin{tabular}{lcc}
\hline \multirow{2}{*}{$\begin{array}{c}\text { Classe/ordem } \\
\text { taxonômica }\end{array}$} & \multicolumn{2}{c}{ Número de indivíduos coletados } \\
\cline { 2 - 3 } Ainhão manso & Laranjeira \\
\hline ARACHNIDA & & \\
Aracneae & 7 & 17 \\
INSECTA & & \\
Chilopoda & 2 & 6 \\
Coleoptera & 5 & 11 \\
Diplopoda & 2 & 2 \\
Diptera & 3 & 8 \\
Hemiptera & - & 2 \\
Hymenoptera & 78 & 143 \\
Lepidoptera & 1 & 2 \\
Orthoptera & 4 & 10 \\
TOTAL & 102 & 201 \\
\hline
\end{tabular}

$\mathrm{Na}$ Figura 1 observa-se que todas as ordens identificadas no solo cultivado com laranjeira exerceram diferença significativa em relação às observadas no cultivo de pinhão manso, exceto diplopoda. Possivelmente, isto ocorreu devido à maior atratividade dos indivíduos pelo aroma característico emitido pelas plantas citrícolas e também à maior quantidade de massa seca depositada no solo durante os períodos de senescência das folhas e de frutos atacados pela mosca das frutas (Ceratitis capitata) - praga identificada na área durante a pesquisa.

No solo cultivado com pinhão manso houve predominância populacional para Hymenoptera > Araneae > Coleoptera > Orthoptera > Diptera > Chilopoda $=$ Diplopoda $>$ Lepdoptera, enquanto que no solo com a cultura da laranjeira prevaleceu a seguinte sequência: Hymenoptera > Araneae > Coleoptera > Orthoptera $>$ Diptera $>$ Chilopoda $>$ Diplopoda $=$ Hemiptera $=$ Lepdoptera.

$\mathrm{Na}$ área implantada com pinhão manso apenas as ordens Araneae e Hymenoptera caracterizaram-se como dominante e superdominante, respectivamente (Tabela 2), enquanto que as demais foram não dominantes; no laranjal, as ordens Aracneae, Chilopoda, Coleoptera, Diptera, Orthoptera classificaram-se como dominantes enquanto a Hymenoptera foi superdominante.

Com relação à abundância, as ordens apresentaram comportamento semelhante em ambos os ambientes, destacando-se Hymenoptera como super dominante e Aracneae como muito dominante. 


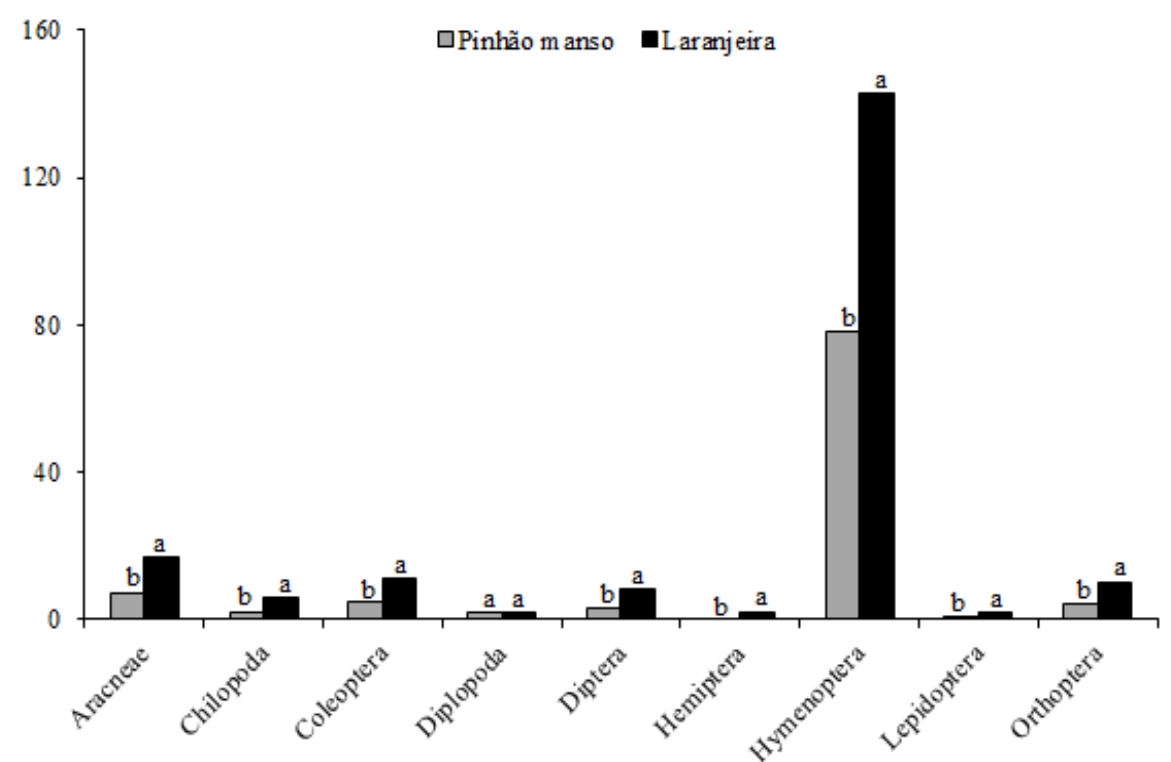

Figura 1. Distribuição do número de indivíduos de acordo com o grupo taxonômico em área com pinhão manso (Jatropha curcas L.) e laranjeira (Citrus sinensis L.).

Tabela 2. Dominância (D) e Abundância (S) de indivíduos coletados em área com pinhão manso (Jatropha curcas L.) e laranjeira (Citrus sinensis $\mathrm{L}$ ).

\begin{tabular}{lcccc}
\hline \multirow{2}{*}{ Classe/ordem taxonômica } & $\mathrm{D}$ & $\mathrm{D}$ & $\mathrm{S}$ & $\mathrm{S}$ \\
\cline { 2 - 5 } ARACHNIDA & Pinhão manso & Laranjeira & Pinhão manso & Laranjeira \\
Aracneae & $\mathrm{D}$ & $\mathrm{D}$ & $\mathrm{ma}$ & $\mathrm{ma}$ \\
INSECTA & & & $\mathrm{c}$ \\
Chilopoda & $\mathrm{ND}$ & $\mathrm{D}$ & $\mathrm{c}$ & $\mathrm{a}$ \\
Coleoptera & $\mathrm{ND}$ & $\mathrm{D}$ & $\mathrm{a}$ & $\mathrm{r}$ \\
Diplopoda & $\mathrm{ND}$ & $\mathrm{ND}$ & $\mathrm{c}$ & $\mathrm{c}$ \\
Diptera & $\mathrm{ND}$ & $\mathrm{D}$ & - & $\mathrm{r}$ \\
Hemiptera & - & $\mathrm{ND}$ & $\mathrm{sa}$ & $\mathrm{r}$ \\
Hymenoptera & $\mathrm{SD}$ & $\mathrm{SD}$ & $\mathrm{r}$ & $\mathrm{c}$ \\
Lepidoptera & $\mathrm{ND}$ & $\mathrm{ND}$ & $\mathrm{c}$ & $\mathrm{c}$ \\
Orthoptera & $\mathrm{ND}$ & $\mathrm{D}$ & $\mathrm{s}$ & $\mathrm{c}$ \\
\hline
\end{tabular}

$\mathrm{ND}=$ Não dominante; $\mathrm{D}=$ Dominante; $\mathrm{SD}=$ Super dominante $\mathrm{r}=$ raro; $\mathrm{d}=$ disperso; $\mathrm{c}=$ comum; $\mathrm{a}=$ abundante $;$ ma $=$ muito abundante; sa $=$ super abundante.

A frequência da macrofauna obteve a mesma tendência nos diferentes solos, exceto para a ordem diplopoda que foi frequente no cultivo de pinhão manso e pouco frequente na laranjeira. As ordens Aracneae e Coleoptera foram classificadas como muito frequentes e os indivíduos hymenopteros como super frequentes. Todas as ordens foram constantes, presentes em mais de $50 \%$ das coletas efetuadas, apesar do baixo número de indivíduos houve uma distribuição semelhante no decorrer das coletas (Tabela 3).

Os índices de diversidade de Shannon e de uniformidade de Pielou estão entre os mais comumente usados e são extremamente apropriados para o uso em ecologia do solo, uma vez que atribuem maiores valores às espécies raras presentes na comunidade (SOUZA et al., 2016); quanto menor o valor do índice de Shannon, menor o grau de incerteza sendo a diversidade da amostra baixa. A diversidade tende a ser mais alta quanto maior o valor do índice. Os valores mais elevados na distribuição dos indivíduos das ordens Hymenoptera, Araneae e Coleoptera (Figura 1) produziram menores índices de diversidade de Shannon $(\mathrm{H})$; no ambiente cultivado com pinhão manso estes índices chegaram a $0,12,1,18$ e 1,32 e para o solo implantado com laranjeira foram de 0,14, 1,07 e 1,26 (Tabela 4), resultados confirmados pelo índice de Pielou alcançando 0,06, 0,58 e 0,65, apresentado-se ainda mais inferior no cultivo de laranjeira $(0,06,0,46$ e 0,66$)$, refletindo diretamente na redução da diversidade. Em trabalho realizado no município de Macaíba-RN com as espécies florestais Acacia mangium, Azadirachta indica e Mimosa caesalpiniaefolia com 12 meses de idade, Silva et al (2014) observaram que a adição de matéria orgânica ao solo elevou os índices de Shannon $(\mathrm{H})$ e de Pielou (e), proporcionando maior diversidade em relação ao solo sob condições naturais de Caatinga. 
Tabela 3. Frequência (F) e Constância (C) de indivíduos coletados em área com pinhão manso (Jatropha curcas L.) e laranjeira (Citrus sinensis L.)

\begin{tabular}{|c|c|c|c|c|}
\hline \multirow{2}{*}{ Classe/ordem taxonômica } & $\mathrm{F}$ & $\mathrm{F}$ & $\mathrm{C}$ & $\mathrm{C}$ \\
\hline & Pinhão manso & Laranjeira & Pinhão manso & Laranjeira \\
\hline \multicolumn{5}{|l|}{ ARACHNIDA } \\
\hline Aracneae & MF & MF & W & W \\
\hline \multicolumn{5}{|l|}{ INSECTA } \\
\hline Chilopoda & $\mathrm{F}$ & $\mathrm{F}$ & W & W \\
\hline Coleoptera & MF & MF & W & W \\
\hline Diplopoda & $\mathrm{F}$ & $\mathrm{PF}$ & W & W \\
\hline Diptera & $\mathrm{F}$ & $\mathrm{F}$ & $\mathrm{W}$ & $\mathrm{W}$ \\
\hline Hemiptera & - & $\mathrm{PF}$ & $\mathrm{W}$ & $\mathrm{W}$ \\
\hline Hymenoptera & SF & SF & W & W \\
\hline Lepidoptera & $\mathrm{PF}$ & $\mathrm{PF}$ & W & W \\
\hline Orthoptera & $\mathrm{F}$ & $\mathrm{F}$ & W & W \\
\hline
\end{tabular}

$\mathrm{PF}$ = pouco frequente; $\mathrm{F}=$ frequente; $\mathrm{MF}$ = muito frequente; $\mathrm{SF}$ - super frequente; $\mathrm{Z}=$ acidental; $\mathrm{Y}=$ acessória; $\mathrm{W}=$ constante

Tabela 4. Índice de Diversidade de Shannon (H) e Índice de Uniformidade de Pielou (e), da macrofauna no cultivo da laranja em Lagoa Seca - PB.

\begin{tabular}{lcccc}
\hline \multirow{2}{*}{ Classe/ordem taxonômica } & $\mathrm{H}$ & $\mathrm{H}$ & $\mathrm{e}$ & $\mathrm{e}$ \\
\cline { 2 - 5 } $\begin{array}{l}\text { ARACHNIDA } \\
\text { Aracneae }\end{array}$ & Pinhão manso & Laranjeira & Pinhão manso & Laranjeira \\
INSECTA & 1,18 & 1,07 & 0,58 & 0,46 \\
Chilopoda & & & & \\
Coleoptera & 1,72 & 1,52 & 0,85 & 0,66 \\
Diplopoda & 1,32 & 1,26 & 0,65 & 0,54 \\
Diptera & 1,72 & 2,00 & 0,85 & 0,86 \\
Hemiptera & 1,54 & 1,40 & 0,76 & 0,86 \\
Hymenoptera & - & 2,00 & - & 0,06 \\
Lepidoptera & 0,12 & 0,14 & 0,06 & 0,86 \\
Orthoptera & 2,02 & 2,00 & 1,00 & 0,56 \\
\hline TOTAL & 1,41 & 1,46 & 0,70 & 5,46 \\
\hline
\end{tabular}

\section{Conclusões}

Os ambientes estudados apresentaram baixa diversidade de indivíduos entomológicos.

O solo cultivado com Citrus sinensis L. apresentou maior abundância e diversidade de ordens da macrofauna edáfica.

As ordens Hymenoptera, Araneae e Coleoptera foram as mais abundantes, dominantes e frequentes, apresentando menores índices de Shannon $(\mathrm{H})$ e de Pielou (e), demonstrando a máxima dominância destas e consequentemente baixa diversidade da amostra.

\section{Referências Bibliográficas}

ALMEIDA, M. A. X.; SOUTO, J. S.; ANDRADE, A. P. Sazonalidade da macrofauna edáfica do Curimataú da Paraíba, Brasil. Revista Ambiência, Guarapuava-PR, v. 11, n. 2, p. 393-407, 2015.

ANTONIOLLI, Z. I.; CONCEIÇÃO, P. C.; BÖCK, V.; PORT, O.; SILVA, R. F. Método alternativo para estudar a fauna do solo. Ciência Florestal, Santa Maria-RS, v. 16, n. 4, p. 407-417, 2006
BODENHEIMER, F. S. Precis d'ecologie animale. Paris: Payot, 1955. 315 p.

CORREIA, K. G.; ARAÚJO, K. D.; AZEVEDO, L. G.; BARBOSA, E. A.; SOUTO, J. S.; SANTOS, T. N. A. S. Macrofauna edáfica em três diferentes ambientes na região do agreste Paraibano, Brasil. Engenharia Ambiental, Espírito Santo do Pinhal-SP, v. 6, n. 1, p. 206-213, 2009.

FERREIRA, D. F. Programa SISVAR - programa de análises estatísticas. Lavras-MG: UFLA, 2003.

LAROCA, S.; MIELKE, O. H. H. Ensaio sobre ecologia de comunidade em Sphingidae na Serra do Mar, Paraná-BR, (Lepidoptera). Revista Brasileira de Biologia, Rio de Janeiro-RJ, v. 35, n. 1, p. 1-19, 1975.

LAVELLE, P. Assessing the abundance and role of invertebrate communities in tropical soils: aims and methods. Journal of African Zoology, Scottsville, v. 102, n. 2, p. 275283,1988

LIMA, S. S.; AQUINO, A. M.; LEITE, L. F. C., VELÁSQUEZ, E. E.; LAVELLE, P. Relação entre macrofauna edáfica e atributos químicos do solo em diferentes 
agroecossistemas. Pesquisa Agropecuária Brasileira, Brasília-DF, v. 45, n. 3, p. 322-331, 2010.

LOPES, J. M. S; DÉO, T. F. G; ANDRADE, B. J. M.; GIROTO, M.; FELIPE, A. L. S.; JUNIOR, C. E. I.; BUENO, C. E. M. S.; SILVA, T. F.; LIMA, F. C. C. Importância econômica do citros no Brasil. Revista Científica de Agronomia, Garça-SP, v. 2, n. 2, p. 1-4, n. 20, 2011.

MARQUES, D. M.; SILVA, A. B.; SILVA, L. M.; MOREIRA, E. A.; PINTO, G. S. Macrofauna edáfica em diferentes coberturas vegetais. Bioscience Journal, Uberlândia-MG, v. 30, n. 5, p. 1588-1597, 2014.

NÁPOLES, F. A. M.; AZEVEDO, C. A. V.; SOUZA, J. T. A.; SOUZA, G. A.V. S.; MONTENEGRO, F. T.; OLIVEIRA, S. J. C. Crescimento e produção de Jatropha curcas L. em função de adubação orgânica. Revista Tecnologia \& Ciência Agropecuária, João Pessoa-PB, v. 10, n. 2, p. 9-15, 2016.

PASQUALIN, L. A.; DIONÍSIO, J. A.; ZAWADNEAK, M. A. C.; MARÇAL, C. T. Macrofauna edáfica em lavouras de cana-de-açúcar e mata no noroeste do Paraná - Brasil. Semina: Ciências Agrárias, Londrina-PR, v. 33, n. 1, p. 7-18, 2012.

RICKLEFS, R. E. A Economia da Natureza. Rio de JaneiroRJ: Guanabara Koogan, 2003. 542 p.

CPRM. COMPANHIA DE PESQUISA DE RECURSOS MINERAIS - SERVIÇO GEOLÓGICO DO BRASIL. Projeto cadastro de fontes de abastecimento por água subterrânea.
Diagnóstico do município de Lagoa Seca, estado da Paraíba. Recife-PE: Editora CPRM/PRODEM, 2005, 19 p.

SILVA, R. F.; TOMAZI, M.; PEZARICO, C. R.; AQUINO, A. M.; MERCANTE, F. M. Macrofauna invertebrada edáfica em cultivo de mandioca sob sistemas de cobertura do solo. Pesquisa Agropecuária Brasileira, Brasília-DF, v. 42, n. 6 , p. 865-871, 2007.

SILVA, A. C. F.; NÓBREGA, C. C.; ARAÚJO, L. H. B.; PINTO, M. G. C.; SANTANA, J. A. S. Macrofauna edáfica em três diferentes usos do solo. Revista Enciclopédia Biosfera, Goiânia-GO, v. 10, n. 18, p. 21-32, 2014.

SILVEIRA NETO, S.; HADDAD, M. L.; MORAES, R. C. B.; LAI REYES, A. E. ANAFAU - análise faunística. PiracicabaSP, Editora ESALQ, 2005. 38 p. Disponível em: <http:www.esalq.usp.br>. Acesso em: 10 jan. 2016,

SOUZA, J. T. A.; FARIAS, A. A.; FERREIRA, R. C. C.; OLIVEIRA, S. J. C.; CAVALCANTE, L. F.; FIGUEIREDO, L. F.; CORREIA, F. G. Macrofauna edáfica em três ambientes diferentes na região do Cariri Paraibano, Brasil. Revista Scientia Agraria Paranaensis, Marechal Cândido RondonPR, v. 15, n. 1, p. 94-99, 2016.

TERRY, I. M. F.; GONZÁLEZ, L. C.; GALLARDO, M. F.; CAIRO, N. C.; ACOSTA, N. R.; PRADO, R. M. Macrofauna del suelo em cuatro fincas en conversión hacia la producción agroecológica em el Municipio Cruces, Cuba. Centro Agrícola, Villa Clara, v. 42, p. 43-52, 2015. 